\title{
Contextual cuing of visual search does not guide attention automatically in the presence of top-down goals
}

\author{
David Luque $^{1,2}$, Tom Beesley ${ }^{3}$, Sara Molinero ${ }^{1,2}$ and Miguel A. Vadillo ${ }^{1}$ \\ ${ }^{1}$ Universidad Autónoma de Madrid, Spain; ${ }^{2}$ Universidad de Málaga, Spain; ${ }^{3}$ University of \\ Lancaster, UK
}

\section{Author note}

David Luque Dhttps://orcid.org/0000-0002-3457-9204

Tom Beesley (iD https://orcid.org/0000-0003-2836-2743

Sara Molinero (Dhttps://orcid.org/0000-0003-1065-2988

Miguel A. Vadillo (Dhttps://orcid.org/0000-0001-8421-816X

We have no conflicts of interest to disclose. DL was supported by grant PGC2018094694-B-I00 (AEI / FEDER, UE) and grant 2017-T1/SOC-5147 (Comunidad de Madrid). MAV was supported by grants PSI2017-85159-P (AEI / FEDER, UE), 2016-T1/SOC-1395, 2020-5A/SOC-19723 (Comunidad de Madrid) and ES/P009522/1 (ESRC). Correspondence concerning this article should be addressed to David Luque, Departamento de Psicología Básica, Facultad de Psicología, Universidad Autónoma de Madrid, 28049 Madrid, Spain. Email: david.luque@gmail.com 


\begin{abstract}
Visual search is faster when it occurs within repeated displays, a phenomenon known as contextual cuing (CC). CC has been explained as the result of an automatic orientation of attention towards a target item driven by learned distractor-target associations. In three experiments we tested the specific hypothesis that CC is an automatic process of attentional guidance. Participants first searched for a T target in a standard CC procedure. Then, they experienced the same repeated configurations (with the T still present), but now searched for a $\mathrm{Y}$ target that was positioned either in a location on the same, or on a different side, from the old T target. Results suggested that there was no interference caused by the old Ttarget: target search was not affected by the relative positions of the $\mathrm{T}$ and $\mathrm{Y}$. Instead, we found a general facilitation in search times for repeated configurations (Experiments 1 and 2). This main effect disappeared when the need for visual search was eliminated in Experiment 3 using a "feature search task". These results suggest that repeated sets of distractors did not trigger an uncontrollable response towards the position of the T; instead, CC was produced by perceptual learning processes.
\end{abstract}

Public Significance Statement: Humans optimize their visual search when they have previous experience with a search display. In this study, we investigate how this search facilitation takes place and if it can be controlled once it is stablished. We found that participants were able to control their attention, which provides evidence against the theory that memory of previous search displays automatically activates learnt configurations of visual search.

Keywords: attention, automaticity, contextual cuing, perceptual learning, visual search 
Looking for the toothpaste is much quicker when you are brushing your teeth in your own bathroom; looking for Waldo is harder the first time you try a new "Where's Waldo?" image. These sketches exemplify the idea that visual search time is reduced when we have had previous experience with a specific visual scenario, a phenomenon known as contextual cuing (CC) (Chun \& Jiang, 1998). In a standard CC experiment, participants look for a target stimulus in a visual display (this target is usually a rotated T: $\vdash$ or $\dashv$ ) among several distractor stimuli (commonly $L$ letters with varied orientations). Once they find the target, participants must respond to its orientation (left or right). On some trials, repeated configurations are presented, in which the Ls and the T are always presented in a set spatial configuration. These repeated trials are interspersed with random configuration trials, in which the Ls and the T are arranged so they form a completely new visual display every trial. In such experiments, visual search times are faster in repeated than in random trials (the CC effect: Chun \& Jiang, 1998; Sisk et al., 2019).

$\mathrm{CC}$ has been thought to reflect the engagement of automatic processes of visual search (Jiang \& Sisk, 2019). Current CC accounts propose that participants learn to associate the position of the distractors with the position of the target when these are both kept constant across trials. Then, when participants perceive a familiar subset of distractors, attention is automatically relocated to the expected position of the target in an efficient, automatic, way (Sisk et al., 2019). Therefore, from this approach CC is produced by automatic guidance of attention triggered by the repeated displays. Following traditional theories on the automaticity of cognitive processes (Shiffrin \& Schneider, 1977), recent experiments have investigated whether $\mathrm{CC}$ might operate in parallel with (and with little interference from) secondary tasks (Manginelli et al., 2013; Pollmann, 2019), and if it can take place when participants are not aware of the distractors-target associations (Colagiuri \& Livesey, 2016; Goujon et al., 2015). In this article we investigated yet another supposed feature of automatic processing, that is, whether CC is uncontrollable. 
The idea that automatic processes are hard to control is based on findings indicating that these processes can interfere with behavior when they are not aligned with current goals (e.g., Stroop, 1935). From this point of view, uncontrollable processes can be characterized as those which interfere with an ongoing task (producing slower RT and/or more errors) even when the participants are trying to inhibit or deactivate them. For example, the Stroop effect demonstrates that word-reading is uncontrollable, because the meaning of the word interferes with the process of naming the font color (your goal), even when the participants are trying to not read the word. There is evidence to suggest that CC might comply with this conceptualization of uncontrollability. Some experiments have shown that once participants have experience with a set of repeated configurations (and CC is already evident), relearning new target positions for the same repeated displays is relatively slow (Makovski \& Jiang, 2010; Zellin et al., 2014). It has been claimed that this effect is produced by the inability of the participants to deactivate the knowledge acquired during the first learning stage, which produces interference during a second (re)learning stage. This hypothesis has been supported by eye-gaze data showing that participants look at the old position of the target first, and then initiate a search process which is very similar to the search path followed in random configurations (Manginelli \& Pollmann, 2009, also see Zinchenko et al., 2020 for similar results from ERP data). Other studies have revealed that $\mathrm{CC}$ decreases as the new target location is farther away from its expected location (Makovski \& Jiang, 2010).

Slow CC relearning can be seen as supportive evidence for an automatic (uncontrollable) nature to $\mathrm{CC}$. However, other results suggest that $\mathrm{CC}$ might be quite flexible. At odds with previous results, Preuschhof et al. (2019) has shown that CC relearning can be very fast-even faster than the first $\mathrm{CC}$ learning. It is not clear why in some experiments relearning is seriously hindered by the first CC learning and in others seems to be facilitated by it. 
Note that the studies reviewed so far were not focused on studying the controllability of CC. Indeed, the speed of CC relearning is not an indicator per se for the (un)controllability of this visual search bias and arguably they could be completely orthogonal. To elaborate, there are factors that affect (re)learning and that have no impact on the level of controllability of the knowledge being learned and vice versa. For instance, learning research has established that learning about familiar stimuli is harder than learning about new stimuli. This effect, known as latent inhibition, might affect how fast participants learn about the repeated configurations in a new stage (Lubow, 1973). Latent inhibition could be produced by a reduction of salience for repeated and therefore well-predicted distractors (McLaren \& Mackintosh, 2000). Supporting this possibility, Ogawa et al. (2007) have shown that a probe at the location of a distractor stimulus is harder to detect within repeated configurations compared to random configurations. This finding might reflect that distractor locations are less salient and/or inhibited in learnt configurations. Consequently, learning new visual-spatial associations from repeated configurations might be hindered because distractors are not fully processed, and the learning rate might therefore be low. This effect would produce slower relearning during the second stage without the need to postulate the operation of any automatic visual search process.

Yet, we can think of another confounding variable pointing to the opposite direction. If distractor-distractor associations are learned during $\mathrm{CC}$ and facilitate the effect, then subsequent CC relearning might be facilitated just because distractor-distractor associations are well-learned after the first stage of CC learning (Beesley et al., 2015). Strong distractordistractor associations could produce faster relearning during a second learning stage but would not inform us about the participation of controlled or automatic processing. To be noted, we are not defending that any of these hypotheses are the true explanation of the relearning results summarized before. Rather, these examples demonstrate that such designs cannot be used to infer levels of controllability, simply because controllability and 
learning rate are independent features of a process, and therefore, they can be affected by independent variables.

In Luque et al. (2017) we set out to investigate CC controllability using a different approach. We used a protocol in which participants first learned a standard CC design in stage 1: they searched for a rotated $T$ among $L$ distractors in repeated configurations and random configurations. Once $\mathrm{CC}$ had been established, participants were then instructed to look for a new letter, a rotated $\mathrm{Y}$, and to ignore the $\mathrm{T}$. Importantly, the same repeated configurations from stage 1 were used in this second stage. The $Y$ target was always placed in one of two possible locations of the display, and the participants were informed about these positions. The question was whether participants could use this information as part of a "top-down" engagement of attention through controlled processes, and therefore inhibit any habitual behavior to search for the old $\mathrm{T}$ target in repeated configurations during stage 2 . The degree of processing of the $T$ was assessed by the degree of response interference produced by that stimulus. Congruent trials were those in which the $T$ and the $Y$ were rotated in the same orientation (and therefore required the same response), while for incongruent trials the two "targets" were oriented in different directions (requiring different responses). Therefore, the response congruency effect indexed to what extent participants were processing the (old target) $\mathrm{T}$, even though this was not the instructed task.

In three experiments, results from the second stage did not show any interaction between the configuration type (repeated vs. random) and the response congruency effect. That is, participants did not process the old T target to any greater degree in repeated configurations compared to in random configurations. These results suggest that participants can effectively deactivate the knowledge acquired in the first stage of the $\mathrm{CC}$ task, and that performance in the $\mathrm{CC}$ task was operating in a controlled manner according to the current set of goals (i.e., to search for the $\mathrm{Y}$ ). However, in Experiments 1 and 2 the new $\mathrm{Y}$ target was always located in the center of the screen. Therefore, there was little need for visual search during the second stage and, arguably, this was not the best scenario for testing automatic 
visual search. For instance, it is possible that automatic guidance to the T was not triggered in this procedure because processing of the distractor configurations was not permitted by the task.

In Luque et al.'s (2017) third experiment, on each trial the $Y$ target was located on either the left or the right side of the screen on the horizontal midline. Thus, participants had to search for the $\mathrm{Y}$ target in the second stage, and this provided a stronger condition in which to test CC automaticity. The $Y$ target was always displayed relatively close to the $T$ target, but farther than parafoveal distance (see Figure 1). In this experiment, search times were faster for repeated than for new configurations; however, this effect did not interact with the response congruency effect, suggesting that participants were not processing the $T$ more in repeated than in random configurations. This result was interpreted as evidence against any automatic orientation towards the $\mathrm{T}$.

This task was designed such that the $T$ and the $Y$ stimuli were always on the same side of the screen, so as to increase the chance of finding a response congruency effect. However, this aspect of the design allows for different interpretations of the main effect of configuration (i.e., faster RTs for repeated configurations than for random configurations). For instance, it is possible that participants learned a new association between the repeated configurations and the consistent placement of the new $Y$ target, and so search for the $Y$ target in repeated configurations was facilitated in this second stage. This possible explanation was originally disregarded because the configuration effect was evident at the very beginning of stage 2. However, Preuschof et al. (2019) have shown that CC "relearning" (learning about a new configuration-target association) can be even faster than the first instance of learning in CC. Moreover, in our experiment, to look for the $\mathrm{T}$ in the second stage was in part beneficial for finding the $\mathrm{Y}$, because both stimuli were always close to each other. This aspect of the design could speed up CC relearning during stage 2, allowing CC relearning even during the first epoch of trials. Therefore, we cannot be sure whether the main effect of configuration was produced by $\mathrm{CC}$ relearning or not. The absence of a 
response congruency effect in Luque et al.'s (2017) Experiment 3 could therefore be explained by the rapid extinction of prior associations, and simultaneous rapid acquisition of new associations for the new $Y$ target. Such an interpretation weakens the claim that contextual cuing is only driven by controlled search processes.

In the present study, we set out to investigate whether the information associated with repeated contexts triggers an automatic guidance of attention towards the $\mathrm{T}$, producing interference when trying to search for a new target in another position. The current studies improved the design of Luque et al. (2017) such that relearning was no longer a potential explanation of the results (Experiments 1 and 2). Also, in Experiment 3, we more directly addressed the mechanisms underlying the configuration effect found in Luque et al. (2017) (and in the current Experiments 1 and 2). In Experiment 3, visual search was eliminated using a "feature search task", which allowed us to assess whether the configuration effect was produced by a facilitation of post-search processing or by a facilitation of visual search.

\section{Experiment 1}

Experiment 1 was similar to the design and procedure of Experiment 3 from Luque et al. (2017), except for the important difference that during the second stage, for each repeated configuration, the $\mathrm{Y}$ target was presented on the same side of the screen as the $T$ for half of all trials, and on the opposite side of the screen for the other half of trials. The two Y locations were also used for trials with random configurations. Therefore, repeated configurations of distractors were not predictive of the position of the $\mathrm{Y}$ target, hindering the learning of new distractor-target associations in this second stage.

Three within-subject factors could modulate search times during the second stage: spatial congruency (indexing if the two targets were displayed at the same or opposite sides of the screen), response congruency (indexing if the two targets required the same response [congruent] or not [incongruent]), and configuration (repeated vs. random) (see Figure 1). Importantly, if repeated configurations produce an automatic activation of search behavior 
towards the T, we would expect to see greater interference effects of spatial congruency and response congruency in the case of repeated configurations compared to random configurations: when the $T$ and the $Y$ are on opposite sides of the screen, search will be slower than when the $T$ and the $Y$ are on the same side of the screen, because attention would be guided to the position of the $\mathrm{T}$ in repeated configurations. For the same reason, responses would be slower if the two targets required different responses than if they required the same response. It is also possible that the effect of these two factors would be additive, such that (for example) the response congruency effect will be larger when the targets are displayed on the same side of the screen.
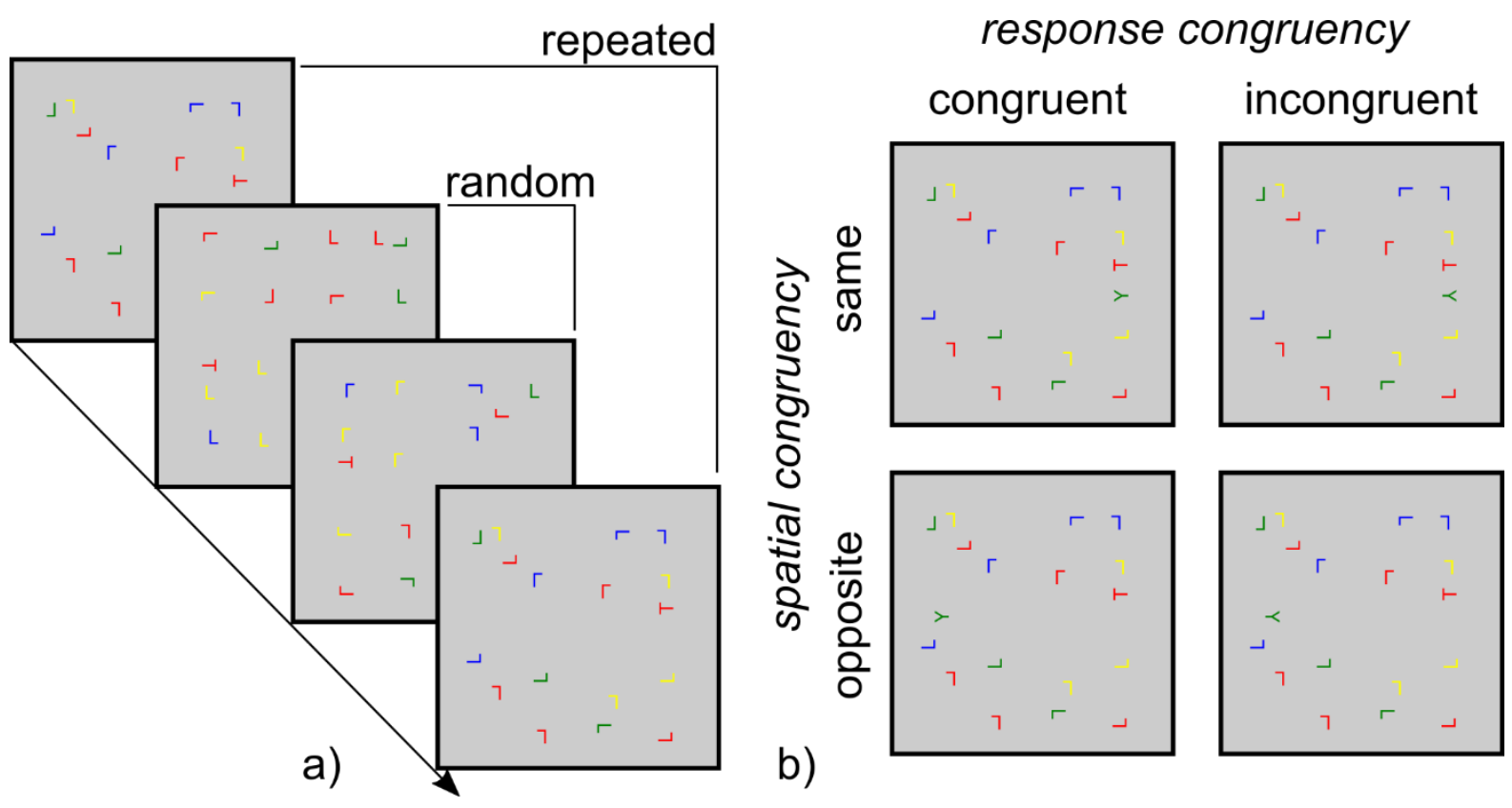

Figure 1. Experimental task. a) Example of a sequence of trials from stage 1, featuring two repeated and two random configurations. b) Examples of the response congruency (different rows) and the spatial congruency (different columns) factors from stage 2 of the experiments. Note that in Experiment 3 all the distractors (Ls) and the T were colored black, and the $\mathrm{Y}$ was colored red.

\section{Method}

Participants. A power analysis showed that a sample size of fifty-two participants would grant statistical power of .80 to detect a small-to-medium size effect of $d_{z}=0.4$. Thus, 
fifty-two participants took part in Experiment 1 (35 women; mean age $=22.53$ ). All participants had normal or corrected to normal vision and completed the experimental task individually in isolated rooms. All participants were students from UNSW Sydney who provided informed consent and received course credit for their participation.

Stimuli. Distractor stimuli were sixteen letter Ls and the target stimulus was a letter T during stage 1 and a letter $Y$ during stage 2. Distractors and targets subtended approximately $0.8^{\circ}$ at a viewing distance of $60 \mathrm{~cm}$. The fixation cross (displayed centrally before each trial) was a $12 \mathrm{~mm}$ square black cross and the background color of the screen was grey. Stimuli were colored blue, red, green or yellow. Distractor stimuli were oriented by rotating the letter $L$ by $0^{\circ}, 90^{\circ}, 180^{\circ}$, or $270^{\circ}$. The position, color, and orientation of distractors were randomly assigned for each configuration, only constrained to have four LS in each quadrant. Target stimuli ( $T$ and $\mathrm{Y}$ ) were oriented by rotating the letters by $90^{\circ}$ or $270^{\circ}$. The program independently assigned the color for the $\mathrm{T}$ - and $\mathrm{Y}$-shaped targets for each participant at random at the start of the task. These colors were retained for the whole experiment. Repeated configurations maintained the same position, color, and orientation of distractor stimuli and the T-shaped target across repetitions. All stimuli (targets and distractors) were positioned in an invisible squared $11 \times 11$ grid, where the central row was reserved only for $Y$ target locations. Each cell of the grid was $50 \times 50$ pixels $(\approx 2.2 \mathrm{~cm})$.

In all trials, T-shaped targets could appear in one of four locations, each in a different quadrant and each target was presented in a different color (randomly assigned for each participant at the start of the task). Hence, the T-shaped target appeared in the same four locations for random and repeated configurations. In the second stage, the Y-target stimulus was added to the configurations used in stage 1. $Y$ targets could appear on either the left or right side of the screen on the horizontal midline (Figure 1). The $\mathrm{Y}$ and $\mathrm{T}$ targets appeared on the same side of the screen on half of the trials during the second stage, and on opposite sides for the remaining trials. The distance between the two targets (the $\mathrm{T}$ and 
the $Y$ ) was one cell when they appeared on the same side of the screen. No distractors were presented on the horizontal midline between the possible positions for the $\mathrm{Y}$ targets.

Procedure. At the start of the experiment, participants read detailed instructions about the visual search task on the computer screen explaining that their goal was to respond to the orientation (pointing left or right) of the $T$ stimulus. Two example displays were presented within the initial instructions. Responses to the target stimulus were made with keys $z$ and $m$ on a standard PC keyboard.

Each trial began with a fixation cross presented in the center of the screen for $1000 \mathrm{~ms}$, and then a configuration of stimuli replaced the fixation cross. Immediately after a correct response, the next trial started. After an incorrect response the word 'ERROR!' appeared in a red font at the center of the screen for $2000 \mathrm{~ms}$. After that, the next trial started after $1 \mathrm{~s}$ of blank ITI. A rest break occurred every 160 trials. The rest time had a maximum duration of $20 \mathrm{~s}$; participants could resume the task at any time during the rest time by pressing the space bar of the keyboard.

Once stage 1 was completed, a new set of instructions was presented on the computer screen before starting stage 2 . The new instructions explicitly stated that now the target stimulus was the letter $Y$ and ignore the $T$. The instructions showed the two possible orientations and the two possible locations of the $Y$ including visual examples. After reading the instructions, participants pressed the space bar to resume the task and stage 2 began.

Stage $1 \mathrm{~A}$ comprised 36 blocks of 4 trials each (all repeated configurations). In stage 1B we introduced random configurations, which comprised half of the trials. Thus, stage 1B comprised 12 blocks of 8 trials ( 4 random and 4 repeated configurations). Stage 2 comprised 8 blocks of 64 trials ( 4 positions of the $T \times 2$ configurations [repeated and random] $\times 2$ positions for the $\mathrm{Y}$ target [left/right] $\times 2$ orientations for the $\mathrm{Y}$ target [left/right] $\times 2$ orientations for the T stimulus [left/right]). 
In both stages, within each block, trials were presented in a random order with the constraint that consecutive trials across adjoining blocks could not present the same repeated configuration.

\section{Results and discussion}

Data preprocessing. The trial-level raw data for all the experiments, along with the scripts used for data preprocessing, are publicly archived at https://osf.io/t2nr4/.

RTs of incorrect responses and RTs greater than 10 seconds were not analyzed. For each participant we calculated the mean RT during Stages 1 and 2 separately. All RTs more than 3 standard deviations above or below the mean RT for that stage were removed from the analysis.

Because repeated configurations were not predictive of the position of the $\mathrm{Y}$ target, relevant learning effects were not expected during the second stage. Considering that, to reduce noise and improve statistical power, blocks were collapsed so the 'block' independent variable was not analyzed. This way, we had the advantage of making ANOVAs simpler. Note that the ANOVA for analyzing data from stage 2 already includes three independent variables. Adding a fourth independent variable would increase the number of tests from 7 to 15 , increasing the probability of making type-I errors (see Cramer et al., 2016 for the risks of using ANOVAs with many factors). We have reported the analyses and figures including block as an independent factor in supplemental materials \#1 (all the experiments). In general, the pattern of results was similar when the factor of block was retained, and, as expected, block did not interact with the other factors in a meaningful way ${ }^{1}$.

\footnotetext{
${ }^{1}$ In Experiment 2, the configuration $\times$ response congruency $\times$ spatial congruency $\times$ block interaction was significant $(p=.047)$. However, this effect did not survive any FWE correction and was not significant in the other experiments (see supplemental materials \#1).
} 
Stage $1 b$. Accuracy of responses was very high $(99.1 \%$, SEM $=0.1)$. We analyzed the $\mathrm{CC}$ effect on RTs during stage $1 \mathrm{~b}$ in which repeated and random configurations were presented. The configuration (repeated vs. random) effect was significant, $t(51)=11.07, p<$ $.001, d_{z}=1.54$, mean RT for repeated configurations $=990.6(\mathrm{SE}=30.62)$, mean RT for random configurations $=1183.1(\mathrm{SE}=29.48)$.

Stage 2. Accuracy of responses was very high $(98.9 \%$, SEM $=0.1)$. We analyzed the impact of stage 1 learning over stage 2 RTs by a 2 (configuration: repeated vs. random) $\times 2$ (response congruency: congruent vs. incongruent) $\times 2$ (spatial congruency: same side of the screen vs. opposite sides of the screen). This ANOVA yielded a main effect of configuration, $F(1,51)=38.33, p<.001, \eta_{\mathrm{p}}^{2}=.43$. This effect of configuration is the consequence of faster responses to repeated configurations (Figure 2). No other main effects or interactions were significant. Among them, the main effect of spatial congruency $\left[F(1,51)=3.37, p=.072, \eta_{\mathrm{p}}^{2}=.06\right]$ and the configuration $\times$ response congruency interaction $\left[F(1,51)=3.45, p=.069, \eta_{\mathrm{p}}^{2}=.06\right]$ were the effects closest to achieving statistical significance.

It is worth noting here that the presence of a strong configuration effect in stage 2 is somewhat surprising. Remember that in stage 2, the $\mathrm{Y}$ targets were presented equally often in the two possible positions on the screen (the left and the right side of the horizontal midline) for each repeated configuration. As such, we have argued that it is unlikely that this effect can be attributed to new learning (a new CC effect) in stage 2 . It is also very unlikely to be driven by a facilitation of target search driven by the distractor-target associations that formed in stage 1 . This is because each repeated configuration was paired with only a single T-target in stage 1, and so even a general search preference for one side of the screen (the side of the predicted T position) that might be established in stage 1, would hinder search for the new $\mathrm{Y}$ target on half of all trials. As such, the strong configuration effect in stage 2 is difficult to explain in terms of enhanced attentional guidance towards an associated spatial location (as it is argued occurs in a typical CC procedure, such as stage 1). One aim of 
Experiment 2 was to replicate this configuration effect (which was successful) and the discussion of the possible causes of this effect is presented after that replication.

Given the theoretical relevance of the configuration $\times$ response congruency interaction (this effect is expected if response times were affected by the orientation of the T more in repeated configurations, compared to random configurations), and that the effect was close to significance levels, we quantified the support for the null using Bayesian statistics. In order to calculate a Bayes Factor (BF) against the specific 2 (configuration) $\times 2$ (response congruency) interaction, we calculated a new dependent response congruency variable (incongruent minus congruent RTs). A Bayesian paired $t$-test was performed on this new variable with configuration as factor (see Luque et al., 2017). In this analysis (as in the equivalent analysis in the following experiments) we tested the null hypothesis (i.e., an equivalent response congruency effect for repeated and random configurations) against the $\mathrm{H} 1$ of a different response congruency effect for repeated and random configurations. $\mathrm{A} \mathrm{BF}_{01}$ larger than 3 is usually considered to reflect substantial support for the null hypothesis and a value larger than 10 strong support. Conversely, values lower than 1/3 are considered substantial evidence for the alternative hypothesis, and values lower than $1 / 10$ strong support for the alternative hypothesis (Wetzels et al., 2011). We used a Cauchy prior with the default values in JASP (scale 0.707 ). The $\mathrm{BF}_{01}$ in favor of the null hypothesis was 1.34. Therefore, our data provided only weak support for the null hypothesis of an absence of a configuration $\times$ response congruency interaction.

We conducted a similar Bayesian analysis for the configuration $\times$ spatial congruency interaction because this effect is expected if visual search was guided by the position of the T more in repeated configurations, compared to random configurations. The $\mathrm{BF}_{01}$ in favor of the null hypothesis was 2.57 ; the evidence provides weak support for the null hypothesis. 
A striking finding from Experiment 1 was the large configuration effect in stage 2. This effect did not interact with the other factors; we found no evidence supporting automatic visual search triggered by the distractors in repeated configurations: search performance was equivalent whether the targets were on the same side or different sides of the screen, and was also equivalent whether the targets required the same response or different responses. This pattern of results dovetails with our previous experiments (Luque et al., 2017) and does not support the automatic guidance of attention account for CC (Jiang \& Sisk, 2019). If the visual search response towards the $T$ was an automatic response (a "search habit", following the terminology used by Jiang \& Sisk, 2019), this automatic response would have produced a configuration $\times$ spatial congruency effect, and likely a configuration $\times$ response congruency effect, because attention would be guided towards the position of the T more often for repeated than random configurations. But we should be cautious with these conclusions. The configuration $\times$ spatial congruency effect was far from significant, but the configuration $\times$ response congruency interaction was close to standard levels of significance and Bayesian analysis did not support the null hypothesis either (i.e., our results did not provide clear evidence for or against the automatic guidance of attention account). Furthermore, the pattern of data in this interaction effect matches the predictions of the automatic guidance of attention account: the response congruency effect (faster responses when the two targets required the same response) was numerically larger for repeated configurations compared to random configurations (see Figure 2) (though this interaction was not significant). Experiment 2 was designed to increase the power to detect these possible effects in order to verify the results from Experiment 1. 

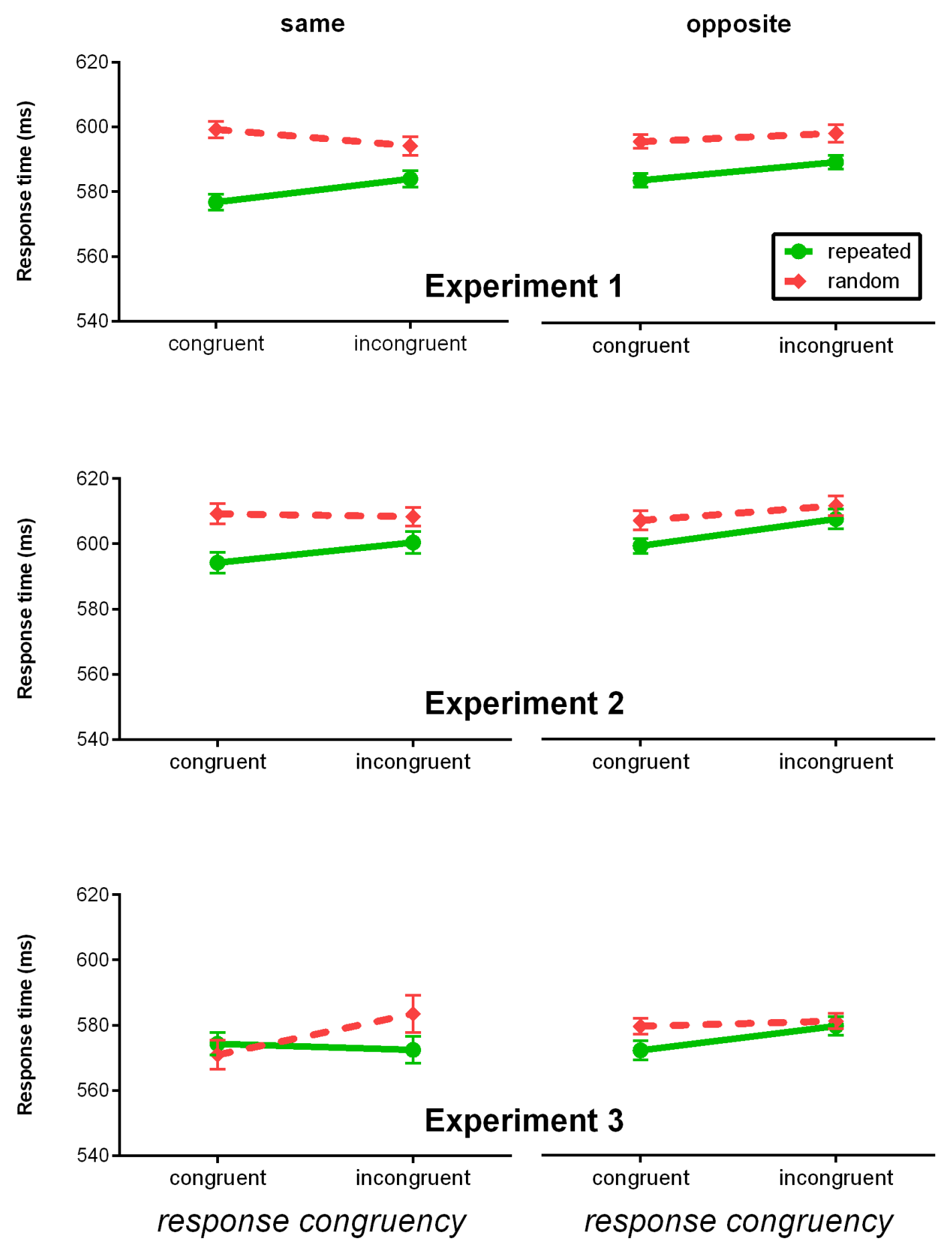

Figure 2. Main results. Second stage mean response times from Experiments 1 to 3 (different rows). In columns, the two levels of the factor spatial congruency: (same vs. different). Response congruency is represented in the $\mathrm{X}$ axis (congruent/incongruent) and configuration in different lines. Error bars indicate within-participant SEM (Cousineau, 2005). 


\section{Experiment 2}

\section{Method}

Participants. Using the configuration $\times$ response congruency effect size from Experiment 1 as a reference $\left(\eta_{\mathrm{p}}^{2}=.06\right)$, a power analysis revealed that achieving a power of .80 would need 121 participants. Due to a limitation on the number of available participants, we recruited a sample of 91 participants ( 57 women; mean age $=21.78$ ), achieving statistical power of .69. However, additional frequentist and Bayesian analyses were conducted which combined the samples of Experiments 1 and 2 in order to increase our chances of revealing evidence for the possible configuration by response congruency and/or configuration by spatial congruency interaction effects (see below).

All participants in Experiment 2 had normal or corrected to normal vision and completed the experimental task individually in isolated rooms. All participants were students from UNSW Sydney who provided informed consent and received course credit for their participation.

Stimuli, procedure, and data preprocessing. The stimuli, procedure and data preprocessing were the same as in Experiment 1 . The only exception is that we reduced the second stage from 8 to 4 blocks, as the block factor did not interact with any relevant independent variable in Experiment 1 (see supplementary material \#1).

\section{Results and discussion}

Stage $1 \mathrm{~b}$. Accuracy of responses was very high $(98.2 \%, \mathrm{SEM}=0.3)$. The configuration effect on RTs was significant, $t(90)=16.22, p<.001, d_{z}=1.70$, mean RT for repeated configurations $=990.1(\mathrm{SE}=25.04)$, mean $\mathrm{RT}$ for random configurations $=1195.3$ $(\mathrm{SE}=26.69)$

Stage 2. Accuracy of responses was very high $(98.2 \%$, SEM $=0.2)$. RTs data for stage 2 are shown in Figure 2. As in Experiment 1, we analyzed the effect of stage 1 
learning over stage 2 RTs by a 2 (configuration) $\times 2$ (response congruency) $\times 2$ (spatial congruency). This ANOVA yielded a main effect of configuration, $F(1,90)=13.51, p<.001$, $\eta_{\mathrm{p}}^{2}=.13$, replicating the pattern seen in Experiment 1 (we return to this finding below). All other effects were not significant (the response congruency effect was the closest $[F(1,90)=$ 3.30, $p=.073, \eta_{\mathrm{p}}^{2}=.04$ ], a consequence of slightly faster RTs for congruent [mean $=602.5$ $(\mathrm{SE}=10.94)]$ than incongruent trials $[$ mean $=607.0(\mathrm{SE}=10.94)$ ]. All other effects produced ps $>.15)$.

As in Experiment 1, we conducted a Bayesian $t$-test assessing whether the response congruency effect was different in repeated and random configurations; the $\mathrm{BF}_{01}$ in favor of the null hypothesis was 5.67, which can be interpreted as substantial support for the null hypothesis. A similar Bayesian analysis for the configuration $\times$ spatial congruency interaction produced a $\mathrm{BF}_{01}$ in favor of the null hypothesis of 4.50 (i.e., substantial support for the null hypothesis).

A combined analysis of the data from Experiment 1 and 2. Experiments 1 and 2 differed in that they were conducted at different times and that stage 2 was shorter in Experiment 2. They were similar in all the remaining aspects, including the pool from which we recruited participants, the laboratory used for conducting the experiment and the researcher in charge of conducting the testing. Given all these similarities, we decided to conduct an additional exploratory analysis which combined the samples from Experiments 1 and 2. We added a factor indexing if the data came from Experiment 1 or 2. This ANOVA only yielded a main effect of configuration, $F(1,141)=38.94, p<.001, \eta_{p}^{2}=.22$, produced by faster RTs for repeated [mean $=615.8(\mathrm{SE}=27.20)$ ] than random configurations [mean $=$ $626.9(\mathrm{SE}=27.20)]$. All other effects produced $p s>.05$. Since the data in Experiment 1 provided only weak support for the null hypothesis of an absence of a configuration $x$ response congruency interaction $\left(\mathrm{BF}_{01}\right.$ in favor of the null hypothesis was 1.34$)$, we further assessed how strong that effect was when pooling the samples from Experiments 1 and 2. A Bayesian $t$-test assessing whether the response congruency effects were different for 
repeated and random configurations yielded a $\mathrm{BF}_{01}=3.30$, that is, substantial support for the null hypothesis. A similar Bayesian analysis for the configuration $\times$ spatial congruency interaction produced $\mathrm{BF}_{01}$ in favor of the null hypothesis of 2.71 .

An automatic guidance of attention account of CC predicts some allocation of attention to the $T$ in repeated configurations, even during stage 2. However, in Experiments 1 and 2, the old target $\mathrm{T}$ did not produce more interference (neither spatial nor response interference) in repeated than in random configurations, failing to support the automaticity claim. Nevertheless, there was a strong main effect of configuration (faster responses for repeated configurations) in stage 2 of both experiments. As we discussed in Experiment 1, we would argue that this effect cannot be attributed to visual search guidance towards the $Y$ in repeated configurations, simply because those configurations did not carry any information about the specific position of the $Y$ (it occurred on the two sides of the screen equally often).

What mechanism might be responsible for this configuration effect in Stage 2? Firstly, it might be produced by a facilitation of response-related processes that happen after the search for the $Y$ has concluded (e.g., response selection would be easier in familiar contexts, Kunar et al., 2007). Sewell et al. (2018) used a version of the Ratcliff (1978) diffusion model to support the notion that visual search is driven primarily by non-search processes. According to this account, visual search reflects the interaction of evidence accumulation and decision thresholds, with the speeded responses on repeated configurations reflecting both that evidence is accumulated at a faster rate and that responses are triggered earlier by lower decision thresholds. Alternatively, the configuration effect might be produced by the perceptual learning of distractor configurations during stage 1 (Beesley et al., 2016; Ogawa et al., 2007; Vadillo et al., 2021). For example, Ogawa et al. have suggested that repeated configurations may lead to inhibition of spatial locations occupied by distractors, which would facilitate search times even without directly driving attention towards a target; search would be facilitated by knowing where not to search, 
rather than by knowing specifically where to search. Experiment 3 sought to test which of these potential processes was most likely to be behind the configuration effect seen in the second stage of Experiments 1 and 2.

\section{Experiment 3}

Experiment 3 used the same fundamental design as Experiments 1 and 2, except that in stage 2, the $\mathrm{Y}$-shaped target was a feature singleton present in red, amongst the distractors and the T target, which were kept in black during the whole experiment. Under this circumstance, the target is known to "pop out" of the display. Such fast feature searches are extremely quick and are not affected by the number of distractor elements in the display. This type of search is driven by a pre-attentive abstraction of the entire visual scene and does not rely on a slow serial item-by-item search (Treisman \& Gelade, 1980). Kunar et al. (2007) demonstrated that even under these fast search conditions, a CC effect can still be obtained, suggesting that the $\mathrm{CC}$ effect can be produced by post-search processes, most likely related to response selection and decision making (see also Schankin \& Schubö, 2009; Sewell et al., 2018). Following the logic of Kunar et al., if the main effect of configuration found in the second stage of the current Experiments 1 and 2 was produced by some sort of facilitation of post-search processing, then the same effect should be observed when a salient feature singleton target is used in Experiment 3. On the other hand, if the effect of configuration was produced by a facilitation of visual search, then this effect should be drastically reduced or eliminated in Experiment 3.

Participants. The effect size for the configuration effect, considering the combined sample of Experiments 1 and 2, was Cohen's $d_{z}=0.51$. Thus, to achieve .80 statistical power we needed at least 33 participants. Thirty-four participants took part in Experiment 3 (32 women; mean age $=19.85)$. All participants had normal or corrected to normal vision and completed the experimental task individually in isolated rooms. All participants were 
students from Autonomous University of Madrid, who provided informed consent and received course credit for their participation.

Stimuli, procedure, and design. All aspects were the same as in Experiment 2, except that all the stimuli were black during the whole experiment apart from the $\mathrm{Y}$, which was red.

\section{Results and discussion}

Stage 1 b. Accuracy of responses was high, as in previous experiments $(98.2 \%$, $\mathrm{SEM}=0.3)$. RT analysis revealed that the effect of configuration was significant, $t(33)=$ $9.63, p<.001$, Cohen's $d_{z}=1.65$, mean RT for repeated configurations $=825.1$ (SE $=$ 28.31), mean $\mathrm{RT}$ for random configurations $=936.6(\mathrm{SE}=32.39)$. It is noteworthy that the visual search times were faster in this experiment than in Experiments 1 and 2, which suggests that visual search was more efficient when all the stimuli were of the same color.

Stage 2. Accuracy of responses was also high during stage $2(97.4 \%, \mathrm{SEM}=0.3)$. We conducted an identical ANOVA to Experiments 1 and 2, which yielded only a main effect of response congruency, $F(1,33)=4.60, p=.039, \eta_{\mathrm{p}}^{2}=.12$, explained by faster responses in congruent than incongruent trials (Figure 2). None of the other effects were significant ( $p$ s $>$.08). Importantly, the configuration effect was not significant, $F(1,33)=0.34, p=.564, \eta_{p}^{2}=$ .01. We established the support for the null hypothesis of no difference using a Bayesian $t$ test on the magnitude of configuration effect. This test yielded a $\mathrm{BF}_{01}=4.65$ (substantial support for the null hypothesis). Thus, the use of a feature singleton target eliminated the strong configuration effect obtained in Experiments 1 and 2, suggesting that the configuration effect in those experiments was likely to have been driven by search processes. 


\section{General Discussion}

It is apparent to us that we can allocate our visual attention in a controlled way, for example, when you are looking for a 1-Euro coin among many other coins in your untidy wallet. What may be more surprising is to claim that sometimes visual search can be out of our direct control (Anderson, 2016; Jiang \& Sisk, 2019). The current experiments investigated the controllability of visual search within repeated configurations in a CC procedure. In these experiments, the participants first experienced a standard CC task in which they were required to search for, and respond to, a T-shaped target. In a second stage they were instructed to search for a Y-shaped target, which appeared equally often in two positions on the screen (left and right along the horizontal midline). Importantly, the same repeated configurations were used throughout and the T-shaped target from the first stage was retained. The repeated configurations did not offer any information about the position of the Y-shaped target. The rationale of the experiments was that, if repeated configurations trigger an automatic orientation of attention towards the position of the Ttarget, then we would expect that the position and orientation of this old target to have an interfering effect on responding to the new $\mathrm{Y}$-target. That would lead to more interference from the old target (i.e., larger response and spatial congruency effects) for repeated configurations compared to random configurations. However, we found no evidence for this pattern within our experiments.

It is noteworthy that the absence of a configuration $\times$ response congruency interaction is not simply due to a complete absence of processing of the T-target, since there is some evidence of interference between the two targets in the present study (see also Luque et al., 2017). When the data from all three experiments are combined, the response congruency effect (collapsing all other factors), shows a small but significant effect, $t(176)=$ 2.66, $p=.009$, Cohen's $d_{z}=0.2, \mathrm{BF}_{10}=2.52$; mean $\mathrm{RT}$ for congruent trials $=591.0$ (SE $=$ 7.11), mean RT for incongruent trials $=595.0(\mathrm{SE}=7.17)$. Thus, it seems that participants did process the $T$ target, at least in some trials, which led to slower responses when the $T$ 
and $Y$ targets required different responses, compared to when they required the same response. This could be a result of the T-target being processed as a distractor (at least on some trials) in the course of searching for the $\mathrm{Y}$, or that participants make errors in implementing their top-down goals in the second stage, erroneously falling back on the attentional set they established in stage 1, searching for the $\mathrm{T}$ instead of for the $\mathrm{Y}$. It should be noted that this was a very weak effect $(4 \mathrm{~ms})$, and hence that participants were very effective in limiting the processing of the T. Most importantly, this effect was not modulated by the type of display configuration, and so is not a result of an automatic allocation of attention as a result of processing the repeated configurations.

Perhaps the most surprising result was the strong configuration effect in stage 2 of Experiments 1 and 2, where we observed faster responses for repeated configurations compared to random configurations. As we have mentioned, this effect did not interact with the response congruency or spatial congruency factors. This effect suggests that the CC produced during stage 1 did have an impact on the processing of repeated configurations in stage 2, even though the evidence points towards a controlled search process for the new $Y$ target.

We suggest that this main effect of configuration in stage 2 is driven by perceptual learning of the configuration of distractors in repeated configurations during stage 1 . Withincompound associations, configural learning, and perceptual grouping effects are well-known mechanisms supporting associative learning, and have been extensively studied in other paradigms (e.g., Luque et al., 2013; Melchers et al., 2006; Treisman, 1982). Indeed, such processes have been proposed as relevant for producing (or enhancing) CC (Beesley et al., 2016; Preuschhof et al., 2019; Vadillo et al., 2021). How exactly this leads to faster search times is currently unclear. The finding that this effect was not found in Experiment 3, in which there was efficient search for a feature singleton target, suggests that it affects the process of slow-serial search. Evidence from experiments using the additional singleton task demonstrate that distractors in expected locations are less effective in slowing target 
processing (Wang \& Theeuwes, 2018). Within the CC literature, Ogawa et al. (2007) found that a probe stimulus was harder to detect when it was placed in the location of a distractor in a repeated configuration, which indicates inhibition of the spatial location occupied by expected distractors. It is possible therefore that perceptual learning, supported by distractor-distractor associations or configural learning, leads to a process of spreading inhibition to these spatial locations where repeated distractors are encountered.

Experiment 3 demonstrated that, when the target was presented in a salient unique color, and therefore a fast, parallel search process was engaged, the configuration effect in stage 2 was abolished. We suggest that this speaks against a relevant role of "decision processes" in the stage 2 configuration effect observed in Experiments 1 and 2, and points towards the effect being due to (what we would term perceptual) processes that occur earlier in search. In their diffusion analysis of CC, Sewell et al. (2018) suggested that the evidence accumulation effect for repeated configurations may be a result of the learning of higherorder spatial information about the distractors and their relationship with the target. For example, when the target and adjacent distractors form a singular recognizable feature, the decision becomes easier to make. It is difficult to see how such an account would readily explain the data in Experiments 1 and 2: any configuration that is learnt between the target and the localized distractors in Stage 1 would benefit the processing of the T target, but not the new $Y$ target.

We have claimed throughout this article that we have failed to find supporting evidence for automaticity of search in CC in Experiments 1 and 2. A caveat is probably in order: Although the evidence supports the null hypothesis for the response congruency $x$ configuration and spatial congruency $\times$ configuration interactions, Bayesian analyses indicate that the evidence for the null is not strong in either case. Although our sample sizes were larger than those typically used in visual search research (Vadillo et al., 2020), future research should include sufficiently large samples for achieving unambiguous evidence-a 
principle that should be the norm in visual search experiments (Colagiuri \& Livesey, 2016; Giménez-Fernández et al., 2020).

It should be noted that we cannot claim that visual guidance towards the $T$ is always easy to control during CC. This is the second study that has directly addressed this aspect of CC, and both studies used similar procedures (see Luque et al., 2017); we cannot know how well these results would generalize to other procedures. One factor that might be relevant for triggering an automatic guidance response is the degree of processing for the distractors. In the second stage of the current experiments the $\mathrm{Y}$-target was easy to find, as it was placed in one out of two locations along the horizontal midline (indeed, response times were faster at the beginning of the second stage than the end of stage 1). Alternatively, we could have positioned the Y-target randomly across the display, and have participants search in an unconstrained manner across the display on each trial, as they had performed in the first stage of each experiment. We would argue this method was not ideally suited here for two reasons. Firstly, our aim was to test the impact of cognitive control on the operation of automatic search processes in CC. To this end, we aimed to provide a reasonably simple new search task that could be followed on each trial in stage 2 , and which would act as the goal under these controlled search processes. It was the operation and implementation of this controlled process that we wished to set in competition against the search process established in stage 1. Secondly, placing the new Y-target in a distributed manner would make it more likely that the T-target would be encountered by chance alone. This would mean that, even if search were conducted in a purely controlled manner in the second stage, we would see response competition on many trials. This would be equivalent across repeated and random configurations, but it would add considerable noise to our estimation of the critical response congruency $\times$ configuration interaction. As we have conceded, we do not know the extent to which our procedure is critical for the controlled processes to dominate visual guidance towards the $T$. Future studies may wish to directly manipulate the 
constraints placed on controlled search in order to understand further how controlled processes come to subdue any automatic guidance in CC.

One study that potentially speaks to the question of what conditions are necessary for observing CC in the presence of top-down goals, is that presented by Beesley et al. (2018, Experiment 1). In the "instructed" condition of their experiment, half of the participants searched for a target in one color, with half of the distractors presented in this same color and half presented in a different color. A CC effect was observed in this condition, both in reaction times and the number of fixations, which suggests that the repeating configurations continue to guide attention efficiently even when a top-down control of attention is engaged (i.e., the goal of attending to one out of the two colors). How does this condition compare with the stage 2 conditions of the current experiments? Here, participants were given a specific instruction that the target could appear in one of two locations. This will have considerably reduced the spatial regions that needed to be checked, assuming that participants were able to control their attention perfectly. In comparison, the task instructions in the Beesley et al. condition led to a much weaker restriction on the search process: participants were required to search through possible candidate targets, and in doing so processed many distractors in the display. These data suggest that the role of the context in guiding attention will be determined by the goals of the task. As we have shown here, when the context becomes irrelevant to the search goals, it has no influence in the guidance of attention to the associated target. Under these conditions we continue to see a small but reliable CC effect, but this is not driven by attentional guidance towards a target location. Future research will be needed to fully understand the task conditions that permit the learnt associations to guide attention automatically.

\section{Conclusion}

The current experiments highlight that $\mathrm{CC}$ is most likely the product of several different learning processes and forms of representation. There is evidence that stimulus- 
target associations guide visual search to the position of the target (Makovski \& Jiang, 2010; Manginelli \& Pollmann, 2009). We have shown here that perceptual learning (i.e., distractordistractor or configural learning) can contribute to the CC effect as well (possibly by spreading inhibition to expected distractor locations), in line with previous results (Beesley et al., 2015, 2016; Preuschhof et al., 2019; Vadillo et al., 2021). And yet, response-related factors might add to the net CC effect (Beesley et al., 2018; Kunar et al., 2007; Sewell et al., 2018). We have also shown that one of these processes, the orientation of attention towards the target position because of distractor-target links, can be effectively suppressed by the participants when it interferes with their ongoing goals - this visual search behavior is controllable (at least in our experimental protocols). We do not know to what extent other processes contributing to the $\mathrm{CC}$ effect are (or are not) controllable because these processes are seldom studied in isolation. Future research examining the automatic or controllable nature of CC would be most effective if it sought to isolate the contribution of these processes (for instance, eliminating the need of visual search for studying the automaticity of response-related processes). We also acknowledge that the use of the term "automatic" fails to portray the important nuances within the study of cognitive processes; it would be more constructive if future work in the field sought to study each feature of "automaticity" in isolation (e.g., dependence on working memory resources, controllability, need of awareness) (Moors \& De Houwer, 2006; see Giménez-Fernández et al., 2020; Luque et al., 2020 for a similar approach).

\section{References}

Anderson, B. A. (2016). The attention habit: How reward learning shapes attentional selection. Annals of the New York Academy of Sciences, 1369(1), 24-39. https://doi.org/10.1111/nyas.12957

Beesley, T., Hanafi, G., Vadillo, M. A., Shanks, David. R., \& Livesey, E. J. (2018). Overt attention in contextual cuing of visual search is driven by the attentional set, but not 
by the predictiveness of distractors. Journal of Experimental Psychology: Learning, Memory, and Cognition, 44(5), 707-721. https://doi.org/10.1037/xIm0000467

Beesley, T., Vadillo, M. A., Pearson, D., \& Shanks, D. R. (2015). Pre-exposure of repeated search configurations facilitates subsequent contextual cuing of visual search. Journal of Experimental Psychology: Learning, Memory, and Cognition, 41(2), 348362. https://doi.org/10.1037/xIm0000033

Beesley, T., Vadillo, M. A., Pearson, D., \& Shanks, D. R. (2016). Configural learning in contextual cuing of visual search. Journal of Experimental Psychology. Human Perception and Performance, 42(8), 1173-1185. https://doi.org/10.1037/xhp0000185

Chen, M., Wang, C., Sclodnick, B., Zhao, G., \& Liu, X. (2019). Executive working memory involved in the learning of contextual cueing effect. Experimental Brain Research, 237(11), 3059-3070. https://doi.org/10.1007/s00221-019-05643-7

Chun, M. M., \& Jiang, Y. (1998). Contextual Cueing: Implicit Learning and Memory of Visual Context Guides Spatial Attention. Cognitive Psychology, 36(1), 28-71. https://doi.org/10.1006/cogp.1998.0681

Colagiuri, B., \& Livesey, E. J. (2016). Contextual cuing as a form of nonconscious learning: Theoretical and empirical analysis in large and very large samples. Psychonomic Bulletin \& Review, 23(6), 1996-2009. https://doi.org/10.3758/s13423-016-1063-0

Cousineau, D. (2005). Confidence intervals in within-subject designs: A simpler solution to Loftus and Masson's method. Tutorials in Quantitative Methods for Psychology, 1(1), 42-45. https://doi.org/10.20982/tqmp.01.1.p042

Giménez-Fernández, T., Luque, D., Shanks, D. R., \& Vadillo, M. A. (2020). Probabilistic cuing of visual search: Neither implicit nor inflexible. Journal of Experimental Psychology. Human Perception and Performance. https://doi.org/10.1037/xhp0000852

Goujon, A., Didierjean, A., \& Thorpe, S. (2015). Investigating implicit statistical learning mechanisms through contextual cueing. Trends in Cognitive Sciences, 19(9), 524533. https://doi.org/10.1016/j.tics.2015.07.009 
Jiang, Y. V., \& Sisk, C. A. (2019). Habit-like attention. Current Opinion in Psychology, 29, 65-70. https://doi.org/10.1016/j.copsyc.2018.11.014

Kunar, M. A., Flusberg, S., Horowitz, T. S., \& Wolfe, J. M. (2007). Does Contextual Cueing Guide the Deployment of Attention? Journal of Experimental Psychology. Human Perception and Performance, 33(4), 816-828. https://doi.org/10.1037/00961523.33.4.816

Lubow, R. E. (1973). Latent inhibition. Psychological Bulletin, 79(6), 398-407. https://doi.org/10.1037/h0034425

Luque, D., Flores, A., \& Vadillo, M. A. (2013). Revisiting the role of within-compound associations in cue-interaction phenomena. Learning \& Behavior, 41(1), 61-76.

Luque, D., Molinero, S., Jevtović, M., \& Beesley, T. (2020). Testing the automaticity of an attentional bias towards predictive cues in human associative learning. Quarterly Journal of Experimental Psychology, 73(5), 762-780. https://doi.org/10.1177/1747021819897590

Luque, D., Vadillo, M. A., Lopez, F. J., Alonso, R., \& Shanks, D. R. (2017). Testing the controllability of contextual cuing of visual search. Scientific Reports, 7(1), 1-10.

Makovski, T., \& Jiang, Y. V. (2010). Contextual cost: When a visual-search target is not where it should be. The Quarterly Journal of Experimental Psychology, 63(2), 216225. https://doi.org/10.1080/17470210903281590

Manginelli, A. A., Langer, N., Klose, D., \& Pollmann, S. (2013). Contextual cueing under working memory load: Selective interference of visuospatial load with expression of learning. Attention, Perception, \& Psychophysics, 75(6), 1103-1117. https://doi.org/10.3758/s13414-013-0466-5

Manginelli, A. A., \& Pollmann, S. (2009). Misleading contextual cues: How do they affect visual search? Psychological Research, 73(2), 212-221. https://doi.org/10.1007/s00426-008-0211-1 
McLaren, I. P. L., \& Mackintosh, N. J. (2000). An elemental model of associative learning: I. Latent inhibition and perceptual learning. Animal Learning \& Behavior, 28(3), 211246. https://doi.org/10.3758/BF03200258

Melchers, K. G., Lachnit, H., \& Shanks, D. R. (2006). The Comparator Theory Fails to Account for the Selective Role of Within-Compound Associations in Cue-Selection Effects. Experimental Psychology, 53(4), 316-320. https://doi.org/10.1027/16183169.53.4.316

Moors, A., \& De Houwer, J. (2006). Automaticity: A Theoretical and Conceptual Analysis. Psychological Bulletin, 132(2), 297-326. https://doi.org/10.1037/00332909.132.2.297

Ogawa, H., Takeda, Y., \& Kumada, T. (2007). Probing attentional modulation of contextual cueing. Visual Cognition, 15(3), 276-289.

https://doi.org/10.1080/13506280600756977

Pollmann, S. (2019). Working memory dependence of spatial contextual cueing for visual search. British Journal of Psychology, 110(2), 372-380. https://doi.org/10.1111/bjop.12311

Preuschhof, C., Sharifian, F., Rosenblum, L., Pohl, T. M., \& Pollmann, S. (2019). Contextual cueing in older adults: Slow initial learning but flexible use of distractor configurations. Visual Cognition, 27(5-8), 563-575. https://doi.org/10.1080/13506285.2019.1668516

Schankin, A., \& Schubö, A. (2009). Cognitive processes facilitated by contextual cueing: Evidence from event-related brain potentials. Psychophysiology, 46(3), 668-679. https://doi.org/10.1111/j.1469-8986.2009.00807.x

Sewell, D. K., Colagiuri, B., \& Livesey, E. J. (2018). Response time modeling reveals multiple contextual cuing mechanisms. Psychonomic Bulletin \& Review, 25(5), 16441665. https://doi.org/10.3758/s13423-017-1364-y 
Shiffrin, R. M., \& Schneider, W. (1977). Controlled and automatic human information processing: II Perceptual learning, automatic attending and a general theory. Psychological Review, 84(2), 127-190. https://doi.org/10.1037/0033-295X.84.2.127

Sisk, C. A., Remington, R. W., \& Jiang, Y. V. (2019). Mechanisms of contextual cueing: A tutorial review. Attention, Perception, \& Psychophysics, 81(8), 2571-2589. https://doi.org/10.3758/s13414-019-01832-2

Stroop, J. R. (1935). Studies of interference in serial verbal reactions. Journal of Experimental Psychology, 18(6), 643-662. https://doi.org/10.1037/h0054651

Treisman, A. (1982). Perceptual grouping and attention in visual search for features and for objects. Journal of Experimental Psychology: Human Perception and Performance, 8(2), 194-214. https://doi.org/10.1037/0096-1523.8.2.194

Treisman, A. M., \& Gelade, G. (1980). A feature-integration theory of attention. Cognitive Psychology, 12(1), 97-136. https://doi.org/10.1016/0010-0285(80)90005-5

Vadillo, M. A., Giménez-Fernández, T., Beesley, T., Shanks, D. R., \& Luque, D. (2021). There is more to contextual cuing than meets the eye: Improving visual search without attentional guidance toward predictable target locations. Journal of Experimental Psychology: Human Perception and Performance, 47(1), 116-120. https://doi.org/10.1037/xhp0000780

Vadillo, M. A., Linssen, D., Orgaz, C., Parsons, S., \& Shanks, D. R. (2020). Unconscious or underpowered? Probabilistic cuing of visual attention. Journal of Experimental Psychology: General, 149(1), 160-181. https://doi.org/10.1037/xge0000632

Wang, B., \& Theeuwes, J. (2018). Statistical regularities modulate attentional capture independent of search strategy. Attention, Perception, \& Psychophysics, 80(7), 1763-1774. https://doi.org/10.3758/s13414-018-1562-3

Wetzels, R., Matzke, D., Lee, M. D., Rouder, J. N., Iverson, G. J., \& Wagenmakers, E.-J. (2011). Statistical Evidence in Experimental Psychology: An Empirical Comparison Using 855 t Tests. Perspectives on Psychological Science: A Journal of the 
Association for Psychological Science, 6(3), 291-298.

https://doi.org/10.1177/1745691611406923

Zellin, M., von Mühlenen, A., Müller, H. J., \& Conci, M. (2014). Long-term adaptation to change in implicit contextual learning. Psychonomic Bulletin \& Review, 21(4), 10731079. https://doi.org/10.3758/s13423-013-0568-z 